\title{
Alveolar Wall
}

National Cancer Institute

\section{Source}

National Cancer Institute. Alveolar Wall. NCI Thesaurus. Code C13085.

The alveolar structure that contains alveolar cells type I and II and macrophages. 